\title{
Differences in daily disposable circle lens performance characteristics
}

\author{
Jeffery M Schafer \\ William T Reindel \\ Marjorie J Rah \\ Osbert Chan \\ Lening Zhang
}

Bausch \& Lomb Incorporated, Rochester, NY, USA
Correspondence: Marjorie J Rah

Bausch \& Lomb Incorporated, $1400 \mathrm{~N}$

Goodman St, Rochester, NY 14609, USA

Tel +I 585 4I36397

Email marjorie.rah@bausch.com
This article was published in the following Dove Press journal:

Clinical Optometry

3 April 2014

Number of times this article has been viewed

Purpose: The purpose of this evaluation was to compare the performance characteristics of two cosmetically tinted contact lenses in the circle lens category that differ in lens design, lens material, and pigment print pattern: etafilcon A (1-Day Acuvue Define; Johnson \& Johnson Vision Care) and hilafilcon B (Naturelle; Bausch \& Lomb Incorporated).

Methods: Two hundred Asian subjects (400 eyes) were enrolled in this 1-month parallel, bilateral, randomized study at ten investigative sites. Study lenses were dispensed at a screening/ dispensing visit, and follow-up visits occurred at 2 weeks and 1 month. Lenses were worn on a daily disposable basis. Fit characteristics were evaluated at each visit, and slit-lamp evaluations were completed at each follow-up visit.

Results: Of the 200 patients enrolled, 172 (344 eyes) completed the study. The proportion of eyes with fully centered lenses was statistically significantly higher for the hilafilcon B group at the 2 -week and 1 -month visits, $P<0.05$. Over all visits, $0.6 \%$ of hilafilcon $\mathrm{B}$ eyes demonstrated incomplete corneal coverage, whereas for the etafilcon A group, $8.5 \%$ of eyes demonstrated incomplete corneal coverage and/or edge lift. The proportion of eyes with adequate lens movement was statistically significantly higher for the hilafilcon B group, $P<0.05$. Over all visits, none of the hilafilcon $\mathrm{B}$ eyes was reported to have excessive movement, whereas for etafilcon $\mathrm{A}$ lenses, $10.2 \%$ of eyes were reported to have excessive movement.

Conclusions: Etafilcon A lenses were significantly less likely to be fully centered and significantly more likely to have incomplete corneal coverage and/or edge lift compared with the hilafilcon B lenses.

Keywords: cosmetic contact lens, circle contact lens

\section{Introduction}

Cosmetic soft contact lenses represent a rapidly growing segment of the contact lens market, especially in Asian countries. A recent report of global soft contact lenses prescribed in 2012 showed prescribing percentages of cosmetically tinted soft contact lenses were as high as 22\% in Indonesia, 32\% in Singapore, $40 \%$ in South Korea, and $56 \%$ in Taiwan. ${ }^{1}$ In contrast, cosmetically tinted lenses represented only $1 \%$ of soft contact lens fits in the United States and the United Kingdom. ${ }^{1}$

Within the cosmetically tinted lens segment, there is a growing trend known as circle lenses. Circle lenses are cosmetically tinted contact lenses that make the eyes appear larger because the tint applied to the lens covers not just a portion of the clear cornea, but extends to cover a portion of the conjunctiva. Lens centration is important in order to achieve the desired cosmetic effect with the lenses; however, social networking communities in Korea have identified a phenomenon in which certain circle cosmetic lenses are displaced from centering on the cornea (Figure 1). This phenomenon has been labeled 
by consumers as the "hula effect", because the lens moves around the eye like a hula hoop. ${ }^{2-4}$ Lenses that demonstrate a "hula effect" can detract from the beauty the user is seeking to experience.

With this growing interest in cosmetic lenses intended to enhance the beauty of the eyes, lens design plays an important role in centering the circle pattern over the cornea. Lens material along with lens design parameters such as back optic zone radius, back surface design, overall thickness, edge thickness, back vertex power, and lens diameter can affect the movement and centration of contact lenses. ${ }^{5-7}$ The purpose of this evaluation was to compare the performance characteristics of two cosmetically tinted contact lenses in the circle lens category that differ in lens design, lens material, and pigment print pattern: etafilcon A (1-Day Acuvue Define; Johnson \& Johnson Vision Care, Jacksonville, FL, USA) and hilafilcon B (Naturelle; Bausch \& Lomb Incorporated, Rochester, NY, USA).

\section{Methods}

Two hundred Asian subjects (400 eyes) were enrolled in this 1-month parallel, bilateral, randomized study at ten investigative sites in Asia and the United States (one site in Hong Kong, two sites in Taiwan, and seven sites in the United States). All subjects were adapted wearers of soft contact lenses and wore a lens in each eye, and each of the subjects' two lenses were of the same manufacture and brand. Additional inclusion criteria were: subjects had to require plano or myopic correction, require contact lens correction from plano to -6.00 diopters in both eyes, and have vision that was correctable through spherocylindrical refraction to 32 letters $(0.3 \log$ MAR $)$ or better in each eye prior to enrolment. Current wearers of 1-Day Acuvue Define Accent (Johnson \& Johnson Vision Care, Jacksonville, FL, USA) or Naturelle (Bausch \& Lomb Incorporated, Rochester, NY USA) lenses were excluded from participation. Following written approved informed consent, one-half of the subjects were randomized to the hilafilcon $\mathrm{B}$ lens and the remaining half was randomized to wear the etafilcon A lens. The study lens parameters are provided in Table 1. Study lenses were dispensed at a screening/dispensing visit, and follow-up visits occurred at 2 weeks and 1 month. Lenses were worn on a daily disposable basis, and lens-fit characteristics and slit-lamp evaluations were completed at each visit.

Distance high-contrast visual acuity with the study lenses was tested at all visits. Visual acuity was measured using a standard logMAR chart (with high [90\%] contrast) with Sloan letters. Visual acuities were converted to Snellen equivalent. Lens centration was assessed as excellent (fully centered), good (slight decentration, no corneal exposure), fair (decentration, intermittent corneal exposure), or poor (incomplete corneal coverage and/or edge lift). Lens movement was assessed as adequate, excessive $(>0.6 \mathrm{~mm})$, insufficient $(<0.2 \mathrm{~mm})$, or adherence. Slit-lamp findings for each eye, including epithelial edema, epithelial microcysts, corneal staining, bulbar injection, limbal injection, upper-lid tarsal conjunctival abnormalities, corneal neovascularization, and corneal infiltrates, were graded for severity on a scale from 0 (no finding) to 4 (severe finding). An adverse event was defined as a sight threatening condition, which may include but was not limited to the following: corneal ulcers, anterior uveitis (iritis), other ocular infections or inflammations, corneal scarring (central $4 \mathrm{~mm}$ ), corneal neovascularization (central $4 \mathrm{~mm}$ ), and/or permanent loss of vision.

\section{Statistical methods}

All statistical comparisons were performed on the null hypothesis that the difference between the lens brands was zero. The $P$-values presented were two-sided and were evaluated using a 0.05 alpha level. Analyses were performed on all eligible, dispensed eyes (completed and discontinued eyes pooled) for efficacy endpoints and for all dispensed eyes (completed and discontinued eyes pooled) for slit-lamp findings. Cochran-Mantel-Haenszel tests were performed in testing the statistical significance of differences in categorical responses with adjustment for study sites. The proportion of eyes with any graded slit-lamp finding greater than grade 2 was computed over all follow-up visits.
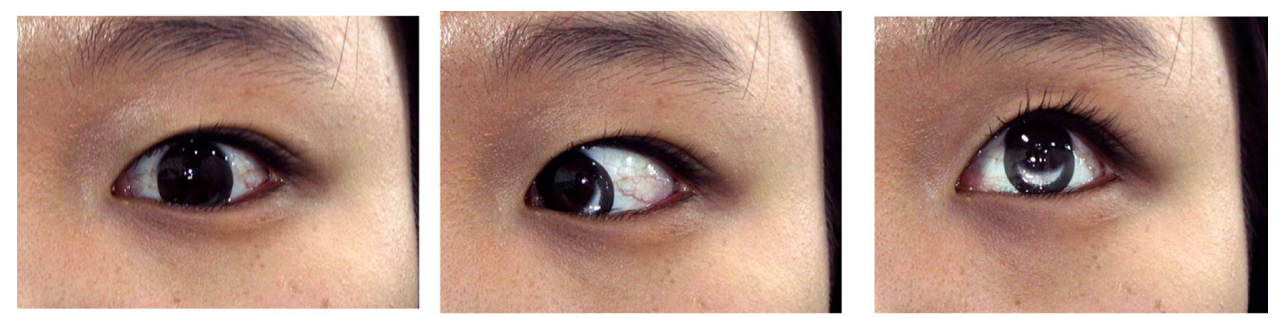

Figure I Cosmetic lens ring pattern with "hula effect" resulting from excess movement. 
Table I Study lens parameters

\begin{tabular}{lll}
\hline Material & Hilafilcon B & Etafilcon A \\
\hline Lens brand & Naturelle & I-Day Acuvue Define \\
Base curve & $8.6 \mathrm{~mm}$ & $8.5 \mathrm{~mm}$ \\
Diameter & $14.2 \mathrm{~mm}$ & $14.2 \mathrm{~mm}$ \\
Power & Plano to -6.00 diopters & Plano to -6.00 diopters \\
Back surface design & Aspheric & Spherical \\
\hline
\end{tabular}

Notes: Naturelle is manufactured by Bausch \& Lomb Incorporated, (Rochester, NY, USA); Acuvue Define is manufactured by Johnson \& Johnson Vision Care (Jacksonville, FL, USA).

\section{Results}

Of the 200 patients enrolled, 172 (344 eyes) completed the study. The mean age of the eligible, dispensed hilafilcon B group was $32.6 \pm 9.5$ years and $32.6 \pm 8.9$ years for the etafilcon A group. All subjects were of Asian descent, and the iris color recorded was predominantly brown ( $98.9 \%$ for the hilafilcon B group and $99.0 \%$ for the etafilcon A group). Baseline demographic data are provided in Table 2.

High-contrast distance visual acuity was $20 / 40$ or better in all eyes for all study visits in both study groups. The mean (standard deviation) average daily wear time, over all follow-up visits, was 12.6 (2.4) hours for the hilafilcon B group and 12.4 (2.4) hours for the etafilcon A group. There were no statistically significant differences in average daily wear time between the groups over all follow-up visits $(P=0.455)$.

Lens centration results at the screening/dispensing visit, and at the 2-week and 1-month follow-up visits are presented in Table 3. The proportion of eyes with fully centered lenses was statistically significantly higher for the hilafilcon $B$ group at the 2 -week and 1 -month visits only, $P<0.05$. Over all visits,

Table 2 Baseline demographic information for the two study groups

\begin{tabular}{|c|c|c|}
\hline & Naturelle & $\begin{array}{l}\text { Acuvue } \\
\text { Define }\end{array}$ \\
\hline Mean age in years (SD) & $32.6(9.5)$ & $32.6(8.9)$ \\
\hline \multicolumn{3}{|l|}{ Sex } \\
\hline Female & $74 \%$ & $76 \%$ \\
\hline Male & $26 \%$ & $24 \%$ \\
\hline \multicolumn{3}{|l|}{ Race } \\
\hline One Japanese parent & $2 \%$ & $4 \%$ \\
\hline Both Japanese parents & $53 \%$ & $57 \%$ \\
\hline Other Asian & $45 \%$ & $39 \%$ \\
\hline $\begin{array}{l}\text { Mean pre-study average daily } \\
\text { wear time in hours (SD) }\end{array}$ & $12.4(3.1)$ & $13.0(3.4)$ \\
\hline Mean sphere in diopters (SD) & $-3.618(1.747)$ & $-3.520(1.639)$ \\
\hline Mean cylinder in diopters (SD) & $-0.334(0.345)$ & $-0.260(0.343)$ \\
\hline
\end{tabular}

Notes: Naturelle is manufactured by Bausch \& Lomb Incorporated, (Rochester, NY, USA); Acuvue Define is manufactured by Johnson \& Johnson Vision Care (Jacksonville, FL, USA).

Abbreviation: SD, standard deviation. only $0.6 \%$ of hilafilcon B eyes demonstrated incomplete corneal coverage, whereas for the etafilcon A group, 8.5\% of eyes demonstrated incomplete corneal coverage and/or edge lift.

Lens movement results at the screening/dispensing visit and at the 2-week and 1-month follow-up visits are presented in Table 4. The proportion of eyes with adequate lens movement was statistically significantly higher for the hilafilcon $\mathrm{B}$ group, $P<0.05$. Over all visits, none of the hilafilcon $\mathrm{B}$ eyes was reported to have excessive movement, whereas for etafilcon A lenses, $10.2 \%$ of eyes were reported to have excessive movement.

Graded slit-lamp findings over all follow-up visits are summarized in Table 5. There were no differences in the proportion of eyes with greater than mild slit-lamp findings. No adverse events were reported for either lens during the study.

\section{Discussion}

Cosmetic "circle lenses" have grown in popularity; however, the combination of a decentered lens with excessive movement can result in consumers experiencing a "hula effect". In the present study, the etafilcon A lenses were less likely to be fully centered and had a greater tendency to have incomplete corneal exposure and/or edge lift. While significantly more etafilcon A lenses exhibited excessive movement, practitioners considered the hilafilcon B lens movement to be adequate. Proper lens assessment is important, as tight or loose fitting soft lenses have been shown to have a more negative impact on ocular physiology than well-fitting lenses ${ }^{6,8}$ Wolffsohn et al ${ }^{6}$ reported that soft lens assessment is typically limited to lens centration and coverage, movement, surface wettability, and subjective comfort and are typically rated as either "good" or "poor". They recommended using a simple diagrammatic representation of lens fit including centration, limbal incursions, horizontal lag, movement on blink in up-gaze, and push-up recovery speed. ${ }^{6}$ In the present study, both centration and movement evaluations were conducted only in the primary gaze position. Future studies should include eye movements over a range of natural viewing conditions to further study the impact on lens movement and corneal coverage.

Lens design plays an important role in creating a successful user experience with these lenses, as movement and centration are affected by such lens parameters as back optic zone radius, back surface design, overall thickness, edge thickness, back vertex power, and lens diameter. ${ }^{5-7}$ Young et $\mathrm{al}^{7}$ showed that lenses with differing back surface designs but similar back 
Table 3 Centration assessments as proportion of eyes

\begin{tabular}{|c|c|c|c|c|c|c|}
\hline & \multicolumn{2}{|c|}{$\begin{array}{l}\text { Screening/dispensing visit } \\
\text { n (\%) }\end{array}$} & \multicolumn{2}{|l|}{$\begin{array}{l}\text { 2-week visit } \\
\text { n (\%) }\end{array}$} & \multicolumn{2}{|c|}{$\begin{array}{l}\text { I-month visit } \\
\text { n (\%) }\end{array}$} \\
\hline & Naturelle & $\begin{array}{l}\text { Acuvue } \\
\text { Define }\end{array}$ & Naturelle & $\begin{array}{l}\text { Acuvue } \\
\text { Define }\end{array}$ & Naturelle & $\begin{array}{l}\text { Acuvue } \\
\text { Define }\end{array}$ \\
\hline Fully centered & I 38 (80.2\%) & I 39 (79.0\%) & 142 (83.5\%) & I 36 (78.2\%) & I 43 (84.1\%) & I 38 (79.3\%) \\
\hline $\begin{array}{l}\text { Slight decentration with } \\
\text { no corneal exposure }\end{array}$ & 25 (14.5\%) & $23(13.1 \%)$ & 14 (8.2\%) & 20 (II.5\%) & 20 (II.8\%) & $24(\mid 3.8 \%)$ \\
\hline $\begin{array}{l}\text { Slight decentration with } \\
\text { intermittent corneal exposure }\end{array}$ & $9(5.2 \%)$ & $9(5.1 \%)$ & I 3 (7.6\%) & $5(2.9 \%)$ & 7 (4.1\%) & $4(2.3 \%)$ \\
\hline $\begin{array}{l}\text { Incomplete corneal coverage } \\
\text { and/or edge lift }\end{array}$ & $0(0 \%)$ & 5 (2.8\%) & I (0.6\%) & I 3 (7.5\%) & $0(0 \%)$ & $8(4.6 \%)$ \\
\hline
\end{tabular}

Notes: Naturelle is manufactured by Bausch \& Lomb Incorporated, (Rochester, NY, USA); Acuvue Define is manufactured by Johnson \& Johnson Vision Care (Jacksonville, FL, USA).

surface sagittal depth do not center the same on the eye. They studied lenses manufactured from the same material while varying one element of the lens design parameters in each group to determine the effect on lens performance. Their results showed that the back surface design (monocurve, bicurve, or aspheric) of soft lenses does have a significant effect on lens fit. Additionally, they reported that decentration tended to increase with increasing radius of curvature, but that lenses of similar primary sagittal depth have similar fitting characteristics when all other aspects of the lens design are the same. They also reported that changes in edge thickness resulted in differences in lens tightness (push-up test) and version lag, and changes in back vertex power resulted in significant differences with horizontal centration, post-blink movement, and overall acceptability. Although the base curve radius and overall diameter of the two lenses tested in the present study are similar, the front/back surface designs of the lenses differ. The hilafilcon B lens has an aspheric front/spherical back surface design, while the etafilcon A lens is designed with spherical front/back surfaces.

The influence of lens material on fitting performance has also been studied. Tranoudis and Efron ${ }^{5}$ studied fit performance using contact lenses of varying material but with the same total diameter, back vertex power, back optic zone radius, and center thickness. Their study showed no significant differences in lens centration, lag, or movement on blink among the materials tested. Wolffsohn et al ${ }^{6}$ have also shown the influence of contact lens material on lens-fit characteristics. They reported differences among materials for vertical centration as well as lag in up and downward gaze and in push-up speed of recovery. Steeper base curves showed a trend toward small lens lag on down-gaze excursion $(P=0.02)$ and a greater movement on blink in up-gaze $(P=0.007)$ compared with flatter lenses; however, these values were not statistically significant after Bonferroni correction for multiple Student's $t$-tests. A significantly faster push-up speed recovery was noted for silicone hydrogels compared with traditional hydrogel lenses $(P<0.05)$. Vertical centration was higher and less central in the hydrogel group than the silicone hydrogel group, with the exception of the Focus DAILIES hydrogel lenses (Alcon Laboratories, Fort Worth, TX, USA) $(P<0.05)$. Although the lenses tested in the present study were both hydrogel lenses, they were of differing materials, as described in Table 1. It is difficult to determine, based on the current study findings, to what extent this may play a role in the fitting characteristics of the two lenses.

Higher order aberrations can also be influenced by poorly centered cosmetically tinted contact lenses. ${ }^{9}$

Table 4 Movement assessments as proportion of eyes

\begin{tabular}{|c|c|c|c|c|c|c|}
\hline & \multicolumn{2}{|c|}{$\begin{array}{l}\text { Screening/dispensing visit } \\
\text { n (\%) }\end{array}$} & \multicolumn{2}{|l|}{$\begin{array}{l}\text { 2-week visit } \\
\text { n (\%) }\end{array}$} & \multicolumn{2}{|c|}{$\begin{array}{l}\text { I-month visit } \\
\text { n (\%) }\end{array}$} \\
\hline & Naturelle & $\begin{array}{l}\text { Acuvue } \\
\text { Define }\end{array}$ & Naturelle & $\begin{array}{l}\text { Acuvue } \\
\text { Define }\end{array}$ & Naturelle & $\begin{array}{l}\text { Acuvue } \\
\text { Define }\end{array}$ \\
\hline Adequate & 170 (98.8\%) & 160 (90.9\%) & I 68 (98.8\%) & 156 (89.7\%) & 169 (99.4\%) & I 58 (90.8\%) \\
\hline Excessive & $0(0 \%)$ & $12(6.8 \%)$ & $0(0 \%)$ & $16(9.2 \%)$ & $0(0 \%)$ & $14(8.0 \%)$ \\
\hline Insufficient & 2 (I.2\%) & $4(2.3 \%)$ & 2 (I.2\%) & 2 (I.I\%) & I (0.6\%) & 2 (I.I\%) \\
\hline Adherence & $0(0 \%)$ & $0(0 \%)$ & $0(0 \%)$ & $0(0 \%)$ & $0(0 \%)$ & $0(0 \%)$ \\
\hline
\end{tabular}

Notes: Naturelle is manufactured by Bausch \& Lomb Incorporated, (Rochester, NY, USA);Acuvue Define is manufactured by Johnson \& Johnson Vision Care (Jacksonville, FL, USA). 
Table 5 Graded slit-lamp findings (n, \%), over all follow-up visits for all dispensed eyes

\begin{tabular}{|c|c|c|c|c|c|c|c|c|c|c|}
\hline \multirow[t]{2}{*}{ Condition } & \multicolumn{5}{|l|}{$\begin{array}{l}\text { Naturelle } \\
\text { n (\%) }\end{array}$} & \multicolumn{5}{|c|}{$\begin{array}{l}\text { Acuvue Define } \\
\text { n (\%) }\end{array}$} \\
\hline & None & Trace & Mild & Moderate & Severe & None & Trace & Mild & Moderate & Severe \\
\hline Epithelial edema & $198(100 \%)$ & 0 & 0 & 0 & 0 & $198(100 \%)$ & 0 & 0 & 0 & 0 \\
\hline Epithelial microcysts & $198(100 \%)$ & 0 & 0 & 0 & 0 & I 97 (99.5\%) & I (0.5\%) & 0 & 0 & 0 \\
\hline Corneal staining & I55 (78.3\%) & $43(21.7 \%)$ & 0 & 0 & 0 & I53 (77.3\%) & 45 (22.7\%) & 0 & 0 & 0 \\
\hline Limbal injection & 177 (89.4\%) & $21(10.6 \%)$ & 0 & 0 & 0 & I 76 (88.9\%) & 22 (II.I\%) & 0 & 0 & 0 \\
\hline Bulbar injection & 170 (85.9\%) & $28(14.1 \%)$ & 0 & 0 & 0 & $169(85.4 \%)$ & $29(14.6 \%)$ & 0 & 0 & 0 \\
\hline $\begin{array}{l}\text { Tarsal conjunctival } \\
\text { abnormalities }\end{array}$ & 164 (82.8\%) & $34(17.2 \%)$ & 0 & 0 & 0 & $160(80.8 \%)$ & $38(19.2 \%)$ & 0 & 0 & 0 \\
\hline $\begin{array}{l}\text { Corneal } \\
\text { neovascularization }\end{array}$ & 188 (94.9\%) & $10(5.1 \%)$ & 0 & 0 & 0 & I 88 (94.9\%) & $10(5.1 \%)$ & 0 & 0 & 0 \\
\hline Corneal infiltrates & $198(100 \%)$ & 0 & 0 & 0 & 0 & $198(100 \%)$ & 0 & 0 & 0 & 0 \\
\hline
\end{tabular}

Notes: Naturelle is manufactured by Bausch \& Lomb Incorporated, (Rochester, NY, USA); Acuvue Define is manufactured by Johnson \& Johnson Vision Care (Jacksonville, FL, USA).

Takabayashi et $\mathrm{al}^{9}$ investigated the differences in ocular wavefront aberrations between 1-Day Acuvue Define daily disposable lenses and clear 1-Day Acuvue lenses. They also looked at the proportion of cosmetically tinted lenses with adequate or poor fit and found that $26 \%$ of 1-Day Acuvue Define lenses had a poor fit. Of the 27 subjects enrolled, 20 were found to have adequate centration and movement on blinking, whereas seven were found to have poor centration or excessive movement on blinking with the cosmetically tinted lenses. The authors reported that total higher order aberrations were approximately 1.5 -fold higher with cosmetically tinted circle lenses that were poorly centered than those of clear and decorative lenses that fit properly, an increase sufficient to decrease quality of vision. The present study results showed that the proportion of eyes with fully centered lenses was statistically significantly higher for the hilafilcon B group compared with the etafilcon A group at the 2-week and 1-month visits.

While the circle patterns of pigment for both the hilafilcon $\mathrm{B}$ and etafilcon $\mathrm{A}$ lenses are intended to enhance the beauty of the eye, they differ in their pattern of pigment. To achieve the cosmetic tint pattern for hilafilcon B lenses, a micro-encapsulation technology that individually encloses color drops is utilized. The etafilcon A lenses seal the colorants between two thin, transparent layers of lens material. Differences in lens centration and encapsulation of pigment did not result in significant differences in slit-lamp findings. Both lenses had similar graded slit-lamp safety profiles.

In conclusion, the etafilcon A lenses were significantly less likely to be fully centered and significantly more likely to have incomplete corneal coverage and/or edge lift compared with the hilafilcon B lenses. Given the cosmetic nature of the lenses, centration and movement are important in achieving the desired cosmetic effect. As circle lenses grow in popularity, practitioners should carefully examine corneal coverage and movement of lenses during different positions of gaze, as lenses that demonstrate a "hula effect" can detract from the cosmetic appearance sought by the user and can potentially decrease quality of vision.

\section{Disclosure}

All authors were employees of Bausch \& Lomb Incorporated at the time of writing this manuscript.

\section{References}

1. Morgan PB, Woods C, Tranoudis IG, et al. International contact lens prescribing in 2012. Cont Lens Spectrum. 2013;28(1):31-44.

2. Korean consumer website blog. Available at: http://blog.naver.com/au rora_b?Redirect $=\log \& \log \mathrm{No}=100157292987$. Accessed January 29, 2014.

3. Korean consumer website blog. Available at: http://blog.naver.com/vcuteb oav?Redirect $=\log \& \log N_{0}=10071236836$. Accessed January 29, 2014.

4. Korean consumer website blog. Available at: http://blog.naver.com/ PostView.nhn?blogId=mirgray\& $\log N_{0}=40103367987$. Accessed January 29, 2014

5. Tranoudis I, Efron N. In-eye performance of soft contact lenses made from different materials. Cont Lens Anterior Eye. 2004;27(3):133-148.

6. Wolffsohn JS, Hunt OA, Basra AK. Simplified recording of soft contact lens fit. Cont Lens Anterior Eye. 2009;32(1):37-42.

7. Young G, Holden B, Cooke G. Influence of soft contact lens design on clinical performance. Optom Vis Sci. 1993;70(5):394-403.

8. Young G, Coleman S. Poorly fitting soft lenses affect ocular integrity. CLAO J. 2001;27(2):68-74

9. Takabayashi N, Hiraoka T, Kiuchi T, Oshika T. Influence of decorative lenses on higher-order wavefront aberrations. Jpn J Ophthalmol. 2013;57(4):335-340. 


\section{Publish your work in this journal}

Clinical Optometry is an international, peer-reviewed, open access journal publishing original research, basic science, clinical and epidemiological studies, reviews and evaluations on clinical optometry. All aspects of patient care are addressed within the journal as well as the practice of optometry including economic and business analyses. Basic and clinical

Submit your manuscript here: http://www.dovepress.com/clinical-optometry-journal

research papers are published that cover all aspects of optics, refraction and its application to the theory and practice of optometry. The manuscript management system is completely online and includes a very quick and fair peer-review system, which is all easy to use. Visit http://www.dovepress. com/testimonials.php to read real quotes from published authors. 